\title{
La construction de la qualité fiable dans les réseaux alimentaires de proximité
}

Building a reliable quality for food products in the context of local alimentary networks

Jean-Louis Vincq, Bernard Mondy et Jean-Pascal Fontorbes

\section{OpenEdition}

Journals

Édition électronique

URL : http://journals.openedition.org/economierurale/2851

DOI : 10.4000/economierurale.2851

ISSN : 2105-2581

Éditeur

Société Française d'Économie Rurale (SFER)

Édition imprimée

Date de publication : 1 septembre 2010

Pagination : 5-19

ISSN : 0013-0559

Référence électronique

Jean-Louis Vincq, Bernard Mondy et Jean-Pascal Fontorbes, «La construction de la qualité fiable dans les réseaux alimentaires de proximité », Économie rurale [En ligne], 318-319 | juillet-octobre 2010, mis en ligne le 01 octobre 2012, consulté le 01 mai 2019. URL : http://journals.openedition.org/ economierurale/2851 ; DOI : 10.4000/economierurale.2851 


\section{La construction de la qualité fiable dans les réseaux alimentaires de proximité}

Jean-Louis VINCQ, Bernard MONDY, Jean-Pascal FONTORBES • UMR Dynamiques Rurales, École nationale de formation agronomique (ENFA), Castanet-Tolosan jean-louis.vincq@educagri.fr, bernard.mondy@educagri.fr, jean-pascal.fontorbes@educagri.fr

D es points de vente collectifs (PVC) sont des lieux de vente situés souvent, mais pas toujours, en zone périurbaine, dans de petits centres ruraux ou sur des exploitations agricoles, aménagés et approvisionnés en tout ou en partie par des producteurs organisés collectivement, et constitués selon des formules juridiques associatives ou sociétaires diverses. Ils font partie de l'ensemble multiforme des « réseaux alimentaires de proximité » dont le développement récent est impressionnant. Comment s'opère le mode de mise en marché des produits alimentaires proposés à la vente? Nous caractériserons tout d'abord les PVC, en précisant leur place au sein des coordinations et leur économie (première partie). Puis, nous verrons comment s'y construit la confiance et à travers quels dispositifs (deuxième partie). Enfin, nous analyserons la façon dont ces dispositifs se combinent pour produire différentes figures symboliques et identifiables, du lien direct producteur-produit (troisième partie).

Notre définition des PVC est large : y sont inclus les points de vente sous enseigne collective, avec franchise, d'origine coopérative ou sociétaire, lesquels connaissent une spectaculaire croissance (Point vert, Gamm Vert par exemple). Nous nous appuyons sur un travail de terrain et une série d'enquêtes réalisées en Midi-Pyrénées ${ }^{1}$ dans plus de la moitié des PVC existants (15 sur 27 en 2007) :

- Auprès de producteurs.

1. Buhler E.-A., Dominique Coquart, Jean-Pascal Fontorbes, Stéphane Girou, Bernard Mondy, Valérie Olivier, Jean Pilleboue, Michaël Pouzenc, Jean-Louis Vincq. (2008). Les relations de proximité agriculteurs-consommateurs : points de vente collectifs et AMAP en Midi-Pyrénées. Sous la direction de Mikael Pouzenc. Toulouse, Dynamiques Rurales. Programme SHS Dossier n ${ }^{\circ}$ 06020615, 265 p.
- Auprès de leurs associés, les entretiens ont surtout porté sur les conditions de création, d'évolution et d'adaptation de ces réseaux. L'accent a été mis sur la constitution du PVC, la distribution des droits de propriété et la participation au travail.

- Auprès des clients, les raisons du choix, le rythme de la fréquentation ont été abordé. Ces enquêtes clients ont été complétées par un questionnaire (45 réponses). L'iconographie a été étudiée et la mise en scène des produits photographiée. Ces données, rapprochées et mises en relation, permettent de construire et de distinguer des figures de PVC.

\section{L'économie des réseaux de proximité}

\section{1. Économie de la qualité et économie de réseau}

Les réseaux alimentaires constituent, au sens de l'approche conventionnaliste, des « environnements » ou des « contextes communs d'interprétation » dans lesquels sont portées et partagées des représentations et des valeurs communes (EymardDuvernay, 2004). Le jugement collectif est une prémisse de l'échange. En établissant les bases d'un classement et d'une évaluation des personnes et des biens, les institutions construisent un principe d'ordre sur lequel les individus s'accordent. Ce sont les conventions ${ }^{2}$. Un accord de marché suppose

2. Le terme désigne "une régularité de comportement $R$ au sein d'une population $P$ telle que ; (1) tous les membres de la population se conforment à $R$; (2) chacun croit que tous les autres membres de $P$ se conforment à $R$ et (3) trouve dans cette croyance une bonne et décisive raison pour se conformer à $R$; (4) par ailleurs, au moins une autre régularité $R$ 'vérifiant les conditions précédentes aurait pu prévaloir »(Orléan, 2004a). 
une convention constitutive, un accord préalable sur la qualité des produits. Lorsque l'évaluation d'un bien est faite par le consommateur, elle est canalisée par une convention marchande. Lorsque l'évaluation est faite par le producteur (c'est le cas du fordisme) la convention est industrielle. Dans la convention industrielle, comme dans la convention domestique également, notent Eymard-Duvernay (2004) ou Salais (2004), c'est le producteur qui détient la capacité de jugement. Dans le cadre des « réseaux de proximité », les biens alimentaires sont mis en marché en situation d'incertitude portant sur la qualité. Mais les produits alimentaires proposés en circuits directs ne sont pas tout à fait assimilables à des biens : les marchés fermiers organisés en réseaux de proximité rompent avec les coordinations marchande et industrielle, ils présentent en effet de nombreuses caractéristiques de services. On parlera ainsi d'aliments-plaisir, aliments-santé, alimentscommodité... Les services sont une manière commode (Eymard-Duvernay, 1986) d'appeler les biens comprenant beaucoup de caractéristiques ou de prestations pour lesquelles la qualité fournie n'est ni complètement, ni clairement définie avant l'acte d'achat et dont la détermination se fait au cours de la transaction. Gadrey définit une activité de services comme une opération qui vise " une transformation d'état, par un prestataire, d'un bien approprié par un utilisateur et qui n'aboutit pas à la production d'un nouveau bien susceptible de circuler économiquement indépendamment $d u$ support» (Gadrey, 1996). C'est bien ce qui se joue dans les réseaux de proximité. Les produits alimentaires en circuit direct se présentent sous la forme de produits incorporant, en quantité importante, des services, c'est-à-dire des particularismes et originalités dans la confection et la fabrication, une livraison régulière et assurée, le goût et la saveur, la garantie, la sécurité et la vocation sanitaires (Hebel, 2005), la fraîcheur... Ce sont des produits dédiés (Knight, 1921), voire « singuliers » (Karpik, 2007). En incorporant des transformations d'état significatives des produits agricoles, les biens alimentaires ainsi proposés sont de plus en plus élaborés. Les produits alimentaires deviennent des aliments-services, c'està-dire des produits qui incorporent des services, essentiellement en assimilant du temps de travail (Sylvander, 1991). Beaucoup de travaux complémentaires soulignent cette évolution vers une incorporation croissante de services dans l'économie des biens agricoles et alimentaires : la tertiarisation des Industries agroalimentaires (IAA) de Néfussi (2005), l'économie de services de Gadrey, (1996), l'économie de la qualité de Karpik (1989), de Gomez (1994), de Allaire (1995), et les idées de service-fonction, serviceexternalité, service-prestation de Aznar, Bretière, Herviou (2006). Dans ce cas, celui d'une évaluation d'un service, et par opposition à une situation où l'évaluation est celle d'un bien, la convention est dite domestique, à condition que l'évaluation soit faite en même temps par le producteur. C'est le cas des AOC ( Delfosse et Letablier, 1995) ou des labels. Mais elle peut être réalisée par le consommateur. La conjonction de l'évaluation (ou d'un jugement) par les consommateurs et celle de services permet de qualifier la convention correspondante de convention de réseau (Eymard-Duvernay, 2004), rejoignant ainsi la théorie du monde connexioniste développée par Boltanski et Chiappello (1999). C'est la situation des produits alimentaires commercialisés en réseaux de proximité. Elle est caractérisée par des liens d'interconnaissance importants, une relation de services forte, une véritable activité des consommateurs (avec le terme, souvent mobilisé, de « consom'acteurs ») qui participent à la construction des principes de jugement. Le consommateur est actif, responsable, il devient citoyen (Dujarier, 2008), producteur (Rochefort, 1997) ou même co-producteur. La consommation est engagée (Delpal, Hatchuel, 2007). Elle porte une dimension politique 
qui permet d'orienter et d'influencer le marché Dubuisson-Quellier et Lamine, 2003). En empruntant la voie de la voice plutôt que celle de l'exit, le client-consommateur présente plusieurs figures actives (Cochoy, 2002).

Les coopérations en vue d'établir l'échange avantageux permettent d'établir une convention partagée exprimant la convergence des intérêts. Pour Salais (2004), la convention est « un système d'attentes réciproques sur les compétences et les comportements, conçu comme allant de soi et pour aller de soi $\gg$ dont Mangematin et Thuderoz (2003) rapprochent la définition de celle de la confiance, en particulier de celle de Fukuyama : "Attente qui naît, au sein d'une communauté, d'un comportement régulier, honnête et coopératif, fondé sur des normes communément partagées, de la part des autres membres de cette communauté». Confiance et convention expriment la même perception des mécanismes coopératifs, à savoir des attentes sur des comportements. Comment sont formalisées ou exprimées ces attentes?

\section{Les logiques d'action au sein des points de vente collectifs}

La première caractéristique de ces marchés est la multiplicité des valeurs qui président à la fabrication et à l'achat. Il y a ainsi plusieurs logiques d'action, terme utilisé pour qualifier l'éventail des rationalités engagées (Karpik, 2007).

3. Ces réseaux reposent sur les principes de jugement associés à la convention de réseau : évaluation et capacité de jugement par le consommateur et caractère de services des biens proposés. Mais d'autres principes sont à l'œuvre, par ex. : ceux de la convention industrielle émergent au sein de cette coordination. Le producteur détient une capacité de jugement, en proposant des produits «faits maison » (sans pour autant parler de fordisme, comme l'autoriserait la convention industrielle pure). L'usage fréquent du mot paysan dans la panneautique et les informations (à côté de «pays ») témoignent de la revendication d'un savoir-faire de la profession.
La multiplicité des arguments des vendeurs/producteurs

La plupart des PVC affichent un (ou des) panneau(x) d'explication souvent tirés de la charte du point de vente qui précise(nt) leurs engagements. Tous portent une revendication d'originalité et de spécificité des produits.

- Le « bien manger » et le « bien boire» sont souvent évoqués : «Un seul objectif défendre "le bien manger" et "le bien boire" »... « C'est pour nous tous le combat bien souvent de toute une vie, un idéal et en prime "la bonne bouffe" ". Dans le même ordre d'idée, une constante revient dans certains PVC, ce sont le goût et les saveurs : avec des expressions comme «les sites du goût »; les « saveurs authentiques des Pyrénées ». Un point de vente affiche: «Nos produits ont du goût. Nous espérons que vous aurez plaisir à les déguster $» . .$. «Alors producteurs, productrices, retroussez encore vos manches, donnez tout au consommateur, en quête du vrai, du bon, de l'authentique goût ».

- Des produits «naturels » sont présentés et mis en évidence avec des panneaux explicatifs. Au-delà des références directes à la nature («produits sains», «sans ajouts »...), on trouve beaucoup d'explications sur les conditions d'élevage telles que : «Poulets fermiers élevés en plein air, nourris au grain $» . .$. «afin de produire des rufs de qualité gustative et sanitaire optimales, nos poules sont élevées en liberté et nourries de céréales pour leur bien être, elles disposent d'une prairie de plus de trois hectares et pondent librement dans des nids garnis de paille. Nous ramassons les aufs chaque jour à la main comme autrefois... ». Le savoir-faire des producteurs est souligné grâce à des photos ou des panneaux explicatifs.

- Une « agriculture paysanne » est parfois revendiquée. C'est un slogan courant. Le discours est nettement plus militant, 
il met en avant un modèle de société dans lequel le maintien d'une agriculture paysanne, souvent associé au respect de l'environnement, serait assuré. S'exprime alors un engagement pour une autre manière de produire, une autre agriculture, voire une autre société.

- Le respect et la préservation de la nature sont mis en avant : "Nous voulons respecter la nature pour respecter votre santé », "Notre bonne vieille terre nous offre "Dame Nature" " ne la décevez pas, respectez-la!» Mais aussi le bien être de l'animal : "Nous sommes prudents dans les méthodes de culture et attentifs au bien être des animaux »... «En achetant dans ce magasin vous participez au maintien et au développement d'une agriculture paysanne locale respectueuse de l'environnement à échelle humaine ». On peut trouver des affiches contre les OGM.

- Le soutien aux agricultures des pays en développement est clairement affiché et mis en valeur. De nombreux PVC possèdent un rayon de commerce équitable plus ou moins important et, souvent, présentent un panneau pédagogique pour expliquer ce qu'est le commerce équitable. Il est souvent associé à un discours militant : «Une agriculture solidaire »... «Consommer équitable, un prix juste pour faire reculer la faim ».... «Nous avons aussi des produits du commerce équitable en solidarité avec d'autres paysans du monde ».

\section{La multiplicité des motifs d'achat}

Les raisons qui guident les clients en PVC apparaissent multiples. Dans une de nos enquêtes (45 personnes interrogées), les usagers d'un PVC mettent en avant, à égalité, le souci de la qualité (le goût et la saveur pour un tiers des réponses) et le soutien aux producteurs (un tiers des attitudes également). La commodité (proximité et facilité d'accès) est également citée de manière significative. La recherche de produits rares, particuliers, uniques, est aussi une raison de l'assiduité dans la fréquentation des lieux de vente. L'achat de produits du commerce équitable relève également de cette logique de soutien. Le prix est un motif d'achat, mais peu cité. Les prix ne viennent qu'en dernière position dans une liste de cinq critères proposés. Nous le voyons, il y a beaucoup de raisons avancées pour fréquenter le PVC. Ils sont repris pour une bonne part, mais pas intégralement, par les producteurs. Il n'y a donc pas de coïncidence.

Les motifs avancés par les producteurs et les consommateurs convergent même s'ils ne coïncident pas toujours. L'offre et la demande exprimées ne se recouvrent pas tout à fait, mais, au sein d'un tel réseau marchand, les acteurs présents s'efforcent de penser leurs relations sur un mode d'interconnaissance, de construire un espace identitaire partagé, d'appartenance (Cochoy, 2001). La multiplicité des arguments et des raisons n'aboutit plus à une décision, mais à un jugement (Karpik, 1996). Au-delà des déclarations, des arguments et des promesses faites, comment l'assurer ? Comment se fier aux produits proposés?

\section{Les dispositifs de confiance dans les réseaux alimentaires de proximité}

L'idée contenue dans ces argumentaires de vente est de faire valoir et de distinguer l'origine du produit pour en annoncer la qualité, et de réaliser l'adéquation entre vente et achat. Mais ces raisons avancées et formalisées n'expliquent pas la fidélité à un produit ou un PVC. Car l'information est insuffisante et peu fiable en elle-même. Il faut pour y croire, forger son opinion en assurant son jugement. La relation d'échange ne se satisfait pas, dans le cadre des circuits courts alimentaires, des repères classiques de l'échange, à savoir les prix. En situation d'asymétrie d'information comme celle qui prévaut dans la vente directe de 
produits agricoles, l'échange aboutit à des dysfonctionnements qui finissent par exclure les «bons produits », et même à interdire l'échange comme l'a montré Akerlof (1970) sur le marché des tacots.

L'acte d'achat s'ancre dans un contexte collectif ou interpersonnel qui assure la confiance. La coordination permet de lever l'incertitude sur l'origine des biens au travers d'une délégation, du choix, à des signes ou des repères sur lesquels s'établit un accord régulier et entretenu, une convention. $\mathrm{Ce}$ sont des repères collectifs. Il s'agit d'outiller la confiance. Les dispositifs de jugement qui reposent sur la confiance mettent en œuvre la collecte d'informations, mais il apparaît que les informations portées en particulier par les engagements et les logiques d'action affichées des producteurs sont incomplètes, non fiables, insuffisantes. Le fait de recourir à des dispositifs d'appui au choix permet de s'assurer de la qualité recherchée. Le terme de délégation, parfois utilisé, signifie la reconnaisance d'une autorité (Eymard-Duvernay, 1989 ; Karpik, 1996 ; Dubuisson-Quellier, Neuville, 2003). Dans la chaîne qui va du producteur au produit, existent des stades ou des étapes qui marquent l'établissement de la qualité du produit, au cours desquelles des tiers interviennent. La construction de la confiance passe par le détour de ces tiers. Ainsi, on attribue une partie de la responsabilité du choix à des personnes ou des signes, preuves, indicateurs de la nature et de la qualité espérée d'un produit. On pourrait parler également de gages ou de promesses, comme le dit d'ailleurs un responsable d'un réseau de PVC sous enseigne.

Le modèle fonctionne comme un syllogisme particulier qui fait dériver d'une prémisse vraisemblable ou probable la levée de l'incertitude sur la qualité du produit : le tiers $\mathrm{T}$ assure vraisemblablement et constitue un gage probable d'assurance de la qualité recherchée du produit $\mathrm{P}$ et comme je fais confiance au tiers $\mathrm{T}$, je peux faire confiance au produit $\mathrm{P}$. C'est un enthymème (Aristote).
S'agissant des produits agricoles, la normalisation peut être un moyen de s'assurer de la qualité (Foray, 1995). Il s'agit plutôt d'un dispositif de promesse qui vise à limiter les risques d'opportunisme (Karpik, 1996) ${ }^{4}$. Elle s'applique peu dans le domaine des circuits courts (au contraire des ventes en GMS, à des grossistes ou à l'exportation). Dispositifs de confiance et dispositifs de promesse se superposent. Nous en dégageons au travers des coordinations alimentaires de proximité quatre types ${ }^{5}$. Ces mécanismes ont déjà été abordés ou évoqués et certains ont été étudiés dans d'autres contextes ou avec d'autres outils d'analyse (Dubuisson-Quellier, Neuville, 2003 ; Karpik, 1996, 2007).

- La certification : les signes de qualité fonctionnent par l'assurance de l'origine (Sylvander, op. cit. ; Delfosse, Letablier, 1995).

- La réputation : la fidélité aux manières de produire et la constance des engagements (Kreps, 1990 ; Rallet et Torre, 2004) consacrent la confiance.

- La qualification : la connaissance des manières de produire et plus particulièrement des moyens pour produire sont un gage de la qualité recherchée (EymardDuvernay, 2004).

- La personnalisation : c'est alors l'ethos du producteur, c'est-à-dire les traits et comportements physiques, la corporalité, les dispositions psychologiques (le caractère), les attitudes politiques et les options sociales qui garantissent la nature du produit.

4. Karpik distingue dans un article de 1996 les dispositifs de confiance des dispositifs de promesse. Ceux-ci permettent d'éviter l'opportunisme. Cette distinction a disparu dans l'économie des singularités parue en 2007. On y parle de dispositifs de confiance.

5. En revanche, Karpik (2007) distingue cinq dispositifs de confiance dans un ensemble de situations observées beaucoup plus étoffé : les réseaux, les appellations, les cicérones (critiques et guides), les classements et les confluences (canalisation sur les lieux de vente). 
Ces quatre formes organisationnelles peuvent être mises en évidence et sont convoquées dans les réseaux directs, notamment les PVC.

\section{Certification : la garantie institutionnelle}

Les signes de qualité, garantis par la puissance publique, établissent la confiance et permettent de la maintenir. La certification repose sur la garantie d'une qualité reconnue. C'est la qualité institutionnelle qui ne se construit pas avec le consommateur (Sylvander, 1995). Les signes de qualité sont, en général, peu mobilisés. La confiance ne repose pas essentiellement sur les signes de qualité, comme l'illustre cette formulation recueillie dans un entretien : ce qui compte c'est « savoir ce que l'on a dans l'assiette et d'où ça vient ». On remarque néanmoins quelques trophées et médailles (notamment les médailles du salon de l'agriculture, mais l'on est alors dans un dispositif de classement $^{6}$ ) couronnant la valeur des produits. $\mathrm{La}$ certification $\mathrm{AB}$ est affichée dans la grande majorité des PVC. Jusqu'à $40 \%$ des produits vendus en $\mathrm{AB}$ dans un PVC! Lorsque le PVC est de type coopératif et égalitaire, AOC ou labels ne fonctionnent pas beaucoup. Il y a alors peu d'indications d'origine ou de terroir! Une cliente d'un PVC déclare : «Je ne regarde pas les étiquettes... Je fais confiance... Le bio, c'est comme le fermier, ce qui compte, c'est que ce soit fait par les agriculteurs. » Où l'on retrouve le souci du lien direct

6. Les dispositifs de confiance s'incarnent dans des techniques et des matériels qui les étayent (tableau 1). En PVC et aussi dans les magasins de produits de producteurs, les MPP, assimilés aux PVC, citons : dispositions du règlement intérieur, lieux de production, identités des producteurs, composition des produits, affichage des permanences, calcul du temps des permanences en relation ou non avec le chiffre d'affaires; commerce équitable et localisation, engagements des producteurs, modes d'approvisionnements, conférences, signes de qualité ( $\mathrm{AB}$ essentiellement), dispositions en cas de rupture d'approvisionnement. produit/producteur qui caractérise « l'entrée en PVC »... «Le discours du producteur, c'est pas bio, mais c'est tout comme! »... Dans une association de producteurs PVC qui mettent en vente uniquement leurs propres produits (au commerce équitable près), l'exposition du signe $\mathrm{AB}$ (et même la revendication qui va avec) est moins marquée, comme si la garantie d'origine pouvait se passer du sigle, du logo, ou de la référence « Le label bio n'est pas très important. Ce qui compte, c'est que les produits soient bio. » Mais dans certaines conditions, les clients des PVC éprouvent le besoin de recourir au signe AB pour s'assurer de l'origine.

Lorsque le PVC fonctionne davantage comme une supérette ou une boutique de proximité pour laquelle la confiance n'est pas complètement assurée, l'affichage du logo est plus important et elle est mise en évidence à l'intérieur du point de vente. Comme si le sigle AB apportait une garantie additionnelle et complémentaire de qualité «Les choses ont changé, le bio attire et il est même demandé, de plus en plus » dit un fidèle. Dans certains PVC, des agriculteurs ont noté parfois une relance de la vente grâce au bio.

La certification du produit n'est donc pas une dimension constitutive forte de l'établissement de la confiance en circuits directs (Delfosse, Bernard, 2004). La convention de qualité qui accorde les acteurs des circuits de proximité repose peu sur les signes de qualité et la certification. Extrêmement faible pour l'appellation controlée et le label rouge, elle existe, surtout en label AB (voir Qualification); à un degré moindre, s'agissant de la mention terroir. Mais il ne s'agit plus d'un signe reconnu.

\section{Réputation : la garantie sociétale}

L'achat des produits alimentaires ne fonctionne pas comme celui des voitures d'occasion. Leur fréquence n'est pas du même ordre. La répétition nécessaire des achats alimentaires permet de réajuster les déci- 
sions d'achat. Les réseaux-échange favorisent la construction de la confiance en associant l'image du producteur au produit. Le « modèle de Kreps » (Kreps, 1990) permet de formaliser la situation. Un usager A1 (ou un client) est amené à coopérer avec un fabricant B (ou un prestataire). Ce prestataire rencontre également d'autres usagers, A2, A3... Ai... qui sont en relation. Ces individus Ai doivent choisir ou non de coopérer avec lui. Si B a honoré ses engagements, alors sa réputation est intacte ; si la confiance est entamée, s'il a trahi, alors sa réputation est souillée, son image est dégradée. Si le jeu se répète suffisamment, la solution est claire : B a intérêt à respecter ses promesses et honorer ses engagements. La répétition de l'acte d'échange et de la transaction est gagée sur la confiance en la stabilité du produit. Le passé est le garant de l'échange. Si une opération n'a lieu qu'une seule fois, le risque de duperie est grand, alors s'installe la défiance et le jeu s'interrompt. Si le jeu se répète, la confiance finit par s'établir sur la base d'une menace d'abandon par un des protagonistes. La réputation se construit sur les évènements passés. Le client ou le consommateur est amené à faire des tests ou des expérimentations : «Si c'est bon, on rachète. » Il faut au préalable que le client soit amené sur les lieux d'achat. C'est lorsqu'il est présent qu'il est amené à élargir, parfois, sa gamme d'achats, sur la base des expériences d'achats passés. Il y a alors diffusion de la confiance à une gamme plus large de produits. Un produit particulier sert de gage à tous les autres pour garantir la qualité recherchée. La réputation est transitive.

Ce sur quoi porte la confiance se rapproche ici du produit. De tous les dispositifs, c'est le plus bilatéral au sens où la confiance ne fait pas intervenir, dans un premier temps, de tiers (tierce personne ou tiers dispositif). Mais la réputation ne se résume pas à la fidélité. Les événements passés assurent la confiance sur la base du respect de l'accord par répétition des transactions. Il y a alors nécessité d'un réseau de diffusion de l'information. Il y a, à côté de la répétition par l'usager de ses actes d'achat, un processus de circulation de l'information au sein d'un réseau d'utilisateurs qui fonde la réputation : le bouche à oreille. C'est la confiance-réseau (Karpik, 1996). Le tiers est alors le réseau constitué par les clients ou les usagers du point de vente. Elle peut être transportée au travers de mécanismes visant à constituer un repère collectif. Ainsi, la réputation d'un individu peut être transférée à une entreprise, une organisation, un collectif ou à un autre membre de l'organisation. On parle alors de confiance indirecte (Mangematin, 2003) caractérisée par sa transitivité. C'est un point important au sein d'une coordination réunissant plusieurs producteurs. La connaissance d'un producteur et sa réputation profitent aux autres producteurs. Le tiers est alors constitué par un producteur associé ou le réseau de bouche à oreille.

\section{Qualification : la garantie de l'origine}

Les méthodes de fabrication sont un point sensible de la convention de qualité fermière. Les conditions connues de production constituent un dispositif de confiance. La qualification fait référence à l'idée de marchés d'organisation (Eymard Duvernay, 2004) c'est-à-dire au fait que la connaissance des facteurs de production mis en œuvre sert de gage au repérage de biens de qualité. Les marchés d'organisation sont les marchés dans lesquels la représentation des systèmes productifs est assurée et négociée. On parle également de biens d'origine (Lagrange et Valceschini, 2007). La confiance se construit et se conserve sur le produit au travers de critères perçus ou connus de l'organisation, ici la connaissance de l'exploitation agricole. Des visites organisées, des participations aux travaux également, des démonstrations, des journées d'animation dans les PVC permettent ainsi d'aborder et 
d'exposer le fonctionnement de l'exploitation. Peut-on pour autant parler de connaissance ? Empiriquement, la connaissance de l'exploitation ou de son système de production est souvent invoquée pour justifier la fréquentation d'un PVC. C'est un facteur fort de confiance, de levée de l'incertitude, une façon convaincante de déléguer son choix à une caractéristique attachée à la qualité produite : le système de production et en particulier les facteurs de production. Mais la connaissance est relative, comme l'expriment d'ailleurs les clients des PVC. Elle est formulée de manière ambiguë et contradictoire, car ils tiennent en même temps un discours affirmé de connaissance pointue d'une exploitation, gage de confiance, et d'aveu d'une connaissance approximative et peu approfondie des exploitations impliquées dans le PVC. L'idée de connaissance est complexe : comment se construit la représentation de l'exploitation? Une visite suffit-elle ? Elle est relative à chacun : quels sont les éléments retenus? Comment se fait l'encodage des informations? Visite? Participation aux travaux ? Photos ? Ouïe dire ? Appréciation de personnes proches qui garantissent la bonne conduite technique? Il n'y a pas de confrontation des points de vue pour définir ce qu'il faut retenir. La connaissance et la transparence d'une seule exploitation suffisent et permettent l'assurance de conduites et de pratiques respectueuses $\mathrm{du}$ «bien produire » ou $\mathrm{du}$ «bon produit ». Il faut considérer que la qualification est transitive, comme la réputation.

\section{La personnalisation, la garantie des options et des engagements des producteurs}

C'est une forme de délégation de la confiance pour laquelle les options sociales et économiques et politiques du producteur, son ethos, constituent le support. On définira l'ethos comme la conjonction du caractère et de la corporalité. Il ne s'agit pas d'un accord sur les caractéristiques des produits ou des facteurs de production, mais d'un accord sur les caractéristiques des producteurs réalisé au travers surtout de son appréhension de la consommation alimentaire et même de sa représentation du monde. Le mécanisme de la «personnalisation " fonctionne bien comme un enthymème, c'est-à-dire un syllogisme dialectique fondé sur le probable : il est vraisemblable ou probable que cet individu a des options politiques et sociales proches des miennes. Comme il est producteur, son produit est fiable. La confiance s'établit sur ces bases. Le partage de certaines valeurs diminue le risque lié aux transactions sur le produit. Le tiers est alors constitué par les représentations du producteur vendeur. Le tiers n'est pas seulement la reconnaissance de la technicité ou de la compétence, mais la personnalité du producteur, son engagement civique par exemple : «Une fois que l'on connaît la personne, on peut faire confiance » dit un client. L'attention à l'ethos du producteur permet de construire et valider cette relation de confiance particulière entre producteur et consommateur. L'ethos du producteur est le gage de la qualité cherchée. Celle-ci porte une dimension relationnelle (Hérault-Fournier, Prigent-Simonin, 2005) ou personnelle (Zucker, 1996). La confiance passe par cette épreuve partagée qui participe de la construction reconnue des bonnes pratiques au sein de ces réseaux.

7. Caractère : ensemble de traits psychologiques ; corporalité : ensemble de traits physiques et vestimentaires. La présence du producteur sur les lieux de vente à qui l'on peut s'adresser pour poser des questions et demander des explications est également un gage important. Les agriculteurs ou les salariés qui tiennent les PVC soulignent l'importance du premier contact. Il est très fréquent, disentils, que le client demande des explications, des éclaircissements lors de sa première venue dans le PVC. Même si on ne pose pas de questions sur les produits ou les modes de production, on apprécie d'avoir la possibilité de pouvoir le faire si on en éprouve le besoin. 
L'incertitude sur l'évaluation de la qualité est résolue parce qu'il existe une définition conventionnelle commune de ce qu'est la compétence (Gomez,1994).

Les dispositifs de confiance ${ }^{8}$ poursuivent un même but : alléger le poids du jugement par la délégation. La confiance repose, nous l'avons vu, sur une enquête active faite par le consommateur comportant plusieurs ressorts. Tout d'abord, pour partie, la certification (et aussi, dans le même registre, la normalisation) : la qualité des produits est signalée (étiquettes, dates de fabrication, composition, label bio, méthodes de fabrication...) ; pour partie également sur les options productives, éthiques, sociales, politiques des producteurs ; pour partie encore, sur le résultat de tests ou d'expérimentations de consommation : «Si c'est bon, on rachète »; pour partie enfin sur la connaissance des manières de produire. Aucun de ces dispositifs de confiance ne peut être légitime si le client consommateur n'a pas, avant l'acte d'achat, l'assurance que le lien est établi entre produit et producteur, que le produit est un produit fermier, un produit de producteur. La confiance repose sur l'idée que l'on a affaire à des produits alimentaires sans intermédiaire : «Du producteur au consommateur » ou qu'il «faut éliminer les intermédiaires ». Quelles sont les indications et les informations mentionnées et portées par les produits ou à l'intérieur des locaux de vente et qui soulignent la proximité voire l'identité du produit et du producteur?

\footnotetext{
8. D'autres dispositifs de confiance sont à l'œuvre. Ainsi le cadre du magasin (agencement, éclairage, espace, circulation) établit une confluence, c'est-àdire une technique de canalisation des clients (Karpik, 2007). Il y a ainsi, souvent, un présentoir par producteur, ce qui permet de mieux personnaliser le produit.
}

\section{Les formes du collectif : les coordinations dans la constitution de réseaux}

Les dispositifs de confiance contribuent à établir de manière convaincante un lien étroit et direct entre le produit et le producteur. Cette liaison est en quelque sorte garante des qualités attendues portées par les produits. Elle fiabilise les logiques d'action affichées. On vient pour acheter des produits garantis d'origine fermière, parfois avec le souci d'aider ou de soutenir les agriculteurs dans les circuits traditionnels. «Nos produits viennent directement de nos fermes. » Derrière les produits, les producteurs. Quelles sont les représentations portées par ces magasins en vue d'établir une relation particulière, privilégiée et pérenne avec le client en suscitant sa confiance par l'assimilation de la qualité du produit à la qualité de celui qui le fabrique et/ou le vend ? La demande ne s'adresse plus au produit, d'où l'importance de l'information attachée aux produits qui importe tout autant que leurs caractéristiques objectives (Sauvée et Valceschini, 2003). Ce n'est plus la logique d'achat mais celle de la fréquentation du point de vente qui joue. Comment et sur quelles indications se construit la confiance en un point de vente? Comment est-elle signifiée?

La convention de qualité relève d'une régularité constatée dans les modalités de l'échange. La coordination comprend les dispositifs qui permettent la qualification du produit. Pour ce qui est des réseaux de proximité, il s'agit de la qualité d'un « produit de producteur ». La signification de la qualité au sein des PVC est construite de manière diverse. Elle s'établit, se construit et se garde au travers de coordinations différentes, selon les voies choisies de mise en marché que l'on peut appeler, des « régimes de coordination » (Karpik, 2007). Il existe différents types de PVC selon la manière avec laquelle ce lien produit/producteur est mis en scène et pour lesquels la construction des accords 
varie. La coordination ne fonctionne pas de manière unifiée. On peut dégager, en relation avec les logiques d'action et les dispositifs de confiance, trois types fondamentaux d'images ou de métaphores des produits agricoles proposés, et autant de champs lexicaux qui qualifient l'esprit du point de vente'. Trois ensembles de références peuvent être identifiés : le PVC «paysan », celui de « la ferme », et celui du «terroir » (tableau 1).

\section{Un premier type : "Aux produits paysans "}

Le terme «paysan » est très fréquemment mobilisé et engagé dans l'affichage à l'intérieur du PVC et surtout l'accroche, le nom souvent affiché à l'extérieur du PVC (« Ô saveurs paysannes », « Le panier paysan », «Au marché paysan », tout récemment « La Halle Paysanne », etc.). On pourrait parler de type pur, dans lequel les produits sont vendus par tous les producteurs. Il n'y a pas ou peu de ventes de produits achetés en dehors du groupe de producteurs. Il s'agit surtout de coopératives ou de groupements d'intérêt économique (GIE). On peut trouver néanmoins des SARL, la question est alors celle des invendus (et dans la SARL, il y a possibilité de rendre les invendus). Les producteurs sont présents et sont les vendeurs. La coordination est égalitaire avec la participation aux décisions de chacun. Les produits sont, le plus souvent, à la charge des producteurs et une cotisation ou une marge est prise par la structure pour faire face aux frais de fonctionnement.

Ces PVC de premier type affichent peu, ou de manière peu ostensible, de signes de qualité. La qualité vient de la garantie des conditions de production et du savoir-faire $\mathrm{du}$ producteur qui donnent au produit un « goût unique ». La certification et la standardisation ne sont pas beaucoup mobilisées et mises en œuvre. Le label $\mathrm{AB}$ et

9. « Faire une typologie d'entreprises, c'est faire une typologie des formes de coordination » (EymardDuvernay, 1992). surtout le label «produit équitable » sont présentés, essentiellement pour élargir la gamme de produits et en garantir l'origine lorsqu'il ne s'agit pas de produits élaborés par les producteurs associés.

Ici, le lien direct est matérialisé par la présence du producteur. Il assure chaque semaine une présence d'autant plus importante que le nombre d'associés est faible. Le temps de présence des producteurs est soit égal en travail, soit établi proportionnellement au chiffre d'affaires. Une banque de travail fonctionne souvent de manière plus ou moins formalisée. Le fonctionnement est démocratique avec une réunion régulière (souvent tous les mois).

Il s'agit de produits agricoles proposés d'origine proche. Le nom du village lieu de production est souvent mis en évidence de manière marquée, et affiché parfois sur les produits ou sur les étalages (en plus des mentions exigées pour l'étiquetage légal). Les producteurs sont d'ici, et cela les qualifie. On pourrait dire « du producteur au consommateur » ou « producteurs réunis », expressions que l'on retrouve affichées et mises en évidence dans certains magasins. La personnification y fonctionne bien, car la présence du producteur est constante. La présence planifiée des producteurs est en rapport avec le statut juridique du collectif qui est privilégié, à savoir la coopérative agricole. Ce sont des petits producteurs qui sont par ailleurs souvent présents sur les marchés de plein vent ou dans d'autres PVC, voire des AMAP. Ils n'achètent pas (ou très peu) de produits agricoles à d'autres producteurs que ceux du collectif. Seuls quelques produits équitables sont proposés, qui élargissent la gamme de produits fermiers ${ }^{10}$. Les légumes occupent une place prépondérante dans ce type de PVC, d'ailleurs, ils sont bien mis en évidence. La question des plannings d'approvisionnement des légumes (et des fruits)

10. Les produits équitables sont présentés comme des produits fermiers. Ils renforcent aussi le côté engagé de la démarche. 
est cruciale. Certains PVC de ce type font valoir qu'ils perdent des clients à cause du manque de tels produits, très demandés.

L'engagement militant des producteurs est très marqué : "Il faut se regrouper pour se défendre avec la volonté de s'en sortir collectivement », mais également sans oublier la valorisation : "Mixer une démarche militante avec une véritable logique commerciale. »Celui des clients également : "Il faut soutenir les formules collectives!»

\section{Un deuxième type : "Aux produits de la ferme "}

Ce sont des PVC reposant sur peu de producteurs, avec présence fréquente de salariés. Ces salariés étant parfois des proches ou des membres de la famille. Les produits sont achetés ou mis en dépôt-vente, lorsque l'approvisionnement ne peut se faire dans le milieu proche ou chez les membres du groupe constitutif. La formule sociétaire est le plus souvent la SARL. Pas toujours, car il peut y avoir des effets d'origine lorsque l'essentiel des produits était, au moment de la création du groupement, d'origine locale et fourni par les producteurs du cru. Il peut y avoir alors une formule coopérative (ou associative) aménagée. Les « conditions initiales », liées au début du cycle de vie de l'organisation fermière, exercent des effets sur le cadre juridique. La forme SARL (ou SICA) est obligatoire quand, manifestement, l'activité commerciale est dominante et avérée, et trop dégagée des activités des exploitations agricoles.

Le local se trouve souvent sur une exploitation qui exerce une sorte de leadership, en constituant souvent une grande partie des ventes, en prenant les décisions (ce qui est facilité par le statut de gérant accordé au chef d'exploitation. La garantie de qualité fermière est donnée par la présence d'un producteur, interlocuteur privilégié des consommateurs, et par le fait que la vente a lieu sur ce qui est ou a été une ferme (ou renvoie explicitement à cette idée) d'où la qualification fréquente «ferme », associée au nom du lieu : de "Candie », de «Pibouls », de « Barcelonne du Gers », du «Quié », de « Magnoac »... par opposition aux PVC purs ( $1^{\text {er }}$ type) qui ne sont pas dans une ferme ou une exploitation et qui mobilisent une autre iconographie de l'ordre du « pays », des « paysan(ne)s », et une autre mobilisation, de l'ordre du « ici », du « local », du « proche », du « village ». On peut parler d'identification au sens où le produit est reconnu comme fermier, par le fait qu'il est vendu par un producteur situé très souvent sur son lieu de vie et d'activité. Il y a en quelque sorte, par transitivité, délégation au producteur/vendeur, de la garantie fermière de tout le magasin.

On trouve davantage de références, ce qui manifeste le souci de trouver une gamme large de produits. On y va pour trouver tous les produits fermiers : "Il y a tout ce qu'on peut espérer », dit une consommatrice qui fréquente ce type de PVC. Celui-ci fonctionne alors comme « une superette avec une garantie des producteurs quant à l'origine des produits », dit un client régulier qui y fait son marché sur la route du supermarché. Le bio se développe dans ce type de magasins et concerne un type de clientèle différent des produits fermiers : le PVC fonctionne alors comme un magasin de vente de produits bio. La qualification du produit existe, et elle tend à se développer lorsque l'origine fermière est moins assurée ou lorsque la visibilité fermière est moindre ou atténuée.

Le nombre de fournisseurs peut être très important comparativement au nombre d'adhérents de la structure. Ces PVC utilisent plus fréquemment l'achat directement aux producteurs plutôt que le dépôt vente : «La marge est bien meilleure. » $\mathrm{La}$ marge est, dans ce type de PVC, un indicateur très observé et suivi.

\section{Un troisième type : "Aux produits de terroir »}

Les magasins de producteurs de pays, tels que Gammvert ou Pointvert, en bénéficiant 
de la marque «Sens du terroir ${ }^{11}$ par exemple (onze boutiques en Midi-Pyrénées en 2007, en pleine expansion) constituent un troisième pôle ou type de points de vente collectifs. Sur la période récente, d'autres magasins de producteurs se sont essayés à la vente collective de produits fermiers, sans franchise. Les magasins de producteurs font référence aux produits de terroir : « Lou terroir », « Les produits du terroir », «Les sens du terroir ». Les références (messages, publicités, photos, panneaux) à l'univers idyllique des campagnes sont nombreuses.

L'approvisionnement déborde largement les producteurs locaux en recourant à des produits alimentaires de toute la France et même de l'étranger. Très peu de fruits et légumes sont mis en vente. Mais on compte beaucoup plus de références : parfois plus de 4000 (quatre fois plus que dans un PVC de premier ou de deuxième type). Il y a peu d'interconnaissance des producteurs. La gamme complète de produits est suivie étroitement. C'est la gamme la plus étendue à l'intérieur des PVC. Les produits locaux, ceux des agriculteurs de la zone, sont bien mis en valeur.

La vente a lieu dans les magasins d'approvisionnement en produits agricoles. Ce lieu est identifié, d'une part, par le fait qu'il est un magasin de producteurs agricoles, qu'il sert aux agriculteurs et qu'il est fréquenté par eux, et d'autre part par le fait qu'il est aussi un lieu de vente directe de végétaux d'ornement, fleurs, arbres fruitiers, quincaillerie agricole... Celui qui fréquente ces lieux peut penser que les agriculteurs sont proches et donc qu'il n'y a pas, en quelque sorte, d'intermédiaire : nous sommes bien, ici encore, dans une logique de lien direct $« \mathrm{du}$ producteur au consommateur $»^{12}$.

11. On peut retenir cette définition de « produits de terroir ». Un produit de terroir est un produit de qualité, un produit sain et naturel, un produit de chez nous, fabriqué par des agricultures locaux avec des ingrédients locaux et enfin, un produit qui fait partie de l'histoire et du patrimoine culturel du pays (Nobecourt, 1998).
La formule juridique est très majoritairement la SICA. La forme SICA permet bien sûr d'acheter aux producteurs de la coopérative située en amont (lesquels, ne sont plus responsable de leurs livraisons). Elle permet surtout d'acheter des produits à des noncoopérateurs (dans la limite de $50 \%$ ). Les adhérents et les coopérateurs ne sont pas responsables de la vente et n'y participent pas. La vente est organisée par la coopérative, les producteurs y sont peu associés, ils viennent parfois participer à des animations ; ils se connaissent peu. Ce sont des salariés qui assurent la vente. Ils relèvent de la direction et non pas du conseil d'administration.

Les PVC de troisième type sont souvent très bien situés, visibles, accessibles et jouent l'effet de vitrine. Leur implantation est soigneusement étudiée alors que l'implantation d'un PVC de premier type est souvent un effet d'opportunité (changement d'usage d'un bâtiment par exemple) et que le PVC de deuxième type est installé sur le siège d'une exploitation.

«Nous n'aimons pas parler de circuits courts mais de circuits de proximité », dit un des concepteurs de la formule. Comme dans les PVC de premier type, on y parle de proximité, mais dans le cadre de «complémentarités évidentes entre les circuits longs et les circuits courts ». Ils s'appuient sur les structures existantes : locaux, producteurs, magasins, abattoirs... Ce sont les « racines », comme le dit un responsable de cette structure. Économies d'échelle (la quantité) et économies de gamme (la diversité, avec une volonté de proposer un éventail complet de produits alimentaires et même au-delà de la quincaillerie, des produits horticoles, etc.)

12. Une particularité est à noter pour un PVC, qui a deux vitrines distinctes; l'une avec la mention «Spécialités du terroir » et l'autre avec la mention « Produits fermiers ». Les termes « terroir» et «fermiers » sont ici rapprochés et associés, car on y trouve à la fois des produits de l'exploitation, de la «ferme », mais aussi d'autres producteurs régionaux, du « terroir » qui ne font qu'apporter leurs produits sans être associés véritablement au PVC. 
caractérisent ce type d'organisation. Pour cela, la structure peut s'appuyer sur des réseaux voisins ou cousins, locaux ou nationaux. Ce sont des réseaux collectifs (en plus de celui des producteurs apporteurs) : commerce équitable, sites remarquables du goût, la marque Parc de la Fédération des Parcs Naturels Régionaux de France (FNPNR), le réseau « sens du terroir $»$. Ce qui est important, c'est l'estampille producteurs. Le produit est associé visuellement à son producteur. La vente de produits fermiers est affichée « en direct du producteur $»$. Ce qui compte, c'est le fait qu'il s'agisse de produits faits par tel producteur identifié et individualisé : la photo de chaque producteur est attachée au rayon sur lequel ses produits sont vendus. Puisque le producteur est représenté, le produit est fiable. On peut parler de représentation, (au double sens d'image/photo du producteur et de mise en scène du producteur). On est dans un local agricole et il ne s'agit pas seulement de profiter des infrastructures, comme le dit un responsable, mais d'offrir un cadre permettant d'établir la confiance et l'assurance de produits fermiers. La représentation du producteur est assurée par sa coopérative qui affiche la présence et la proximité géographique et organisationnelle des producteurs.

L'objectif affiché de ces structures est de «faire du durable et associer le consommateur $»$. Cette déclaration d'un responsable est très intéressante dans sa double préoccupation à faire apparaître la qualité du produit (au travers de l'image de durabilité) et de proposer des produits dédiés (à une demande particulière), singuliers ou uniques. Toute demande est alors, pour le producteur, unique et comparable à aucune autre (Salais, 2004).

\section{Conclusion}

La conviction d'acheter un produit ou de fréquenter un $\mathrm{PVC}$ répondant aux critères de la qualité recherchée est difficile à assurer. Les choix sont multiples et à priori non stabilisés. La décision y est remplacée par le jugement (Karpik, 1996) tant les raisons et critères d'achat et de fréquentation sont nombreux. La croyance n'est pas éliminée. Elle est toujours nécessaire. Au sein des coordinations fermières, elle est étayée par des dispositifs et des mécanismes qui permettent l'engagement dans une telle forme de mise en marché. Ces dispositifs sont multiples. Ils permettent d'atténuer l'incertitude et d'orienter les choix. À l'intérieur des PVC, ces dispositifs de confiance qui servent d'appui au jugement fonctionnent de manière complémentaire en favorisant l'assimila$\operatorname{tion}^{13}$ du produit à des mondes chargés de valeurs attribuables et reconnues. Ils participent ainsi à la construction de différents régimes de coordination porteurs d'images symboliques des PVC, qui soulignent le lien direct, voire identitaire, entre le produit et son producteur et l'encodent dans des figures identifiables : le produit «paysan », « fermier », celui « du terroir ${ }^{14}$.

Les réseaux alimentaires de proximité sont un monde beaucoup plus large que celui des PVC. Marchés paysans (et leurs nouvelles formes : marchés de nuit, de pays...), paniers de biens sur internet, AMAP (Associations pour le maintien de l'agriculture paysanne), Points de vente collectifs, magasins de producteurs, boutiques de pays... toutes ces formules constituent une gamme large de coordinations, en plein

13. Voire d'une incorporation des valeurs attachées à l'achat réalisé (Fischler, 1990).

14. Les coordinations mettent en œuvre des dispositifs de confiance dans des proportions variables. Une convention existe rarement à l'état pur. La convention qui caractérise les PVC (avec les autres réseaux de proximité) est bien d'abord une convention de réseau, mais elle est « hybridée », « pluraliste » (EymardDuvernay, 2004) ou « composite » (Thévenot, 1995 ; Sylvander, 1995), elle est mâtinée d'autres grandeurs, marchande, industrielle, domestique qui assurent la différenciation des réseaux. On peut reconnaitre ainsi dans les PVC de type 1 la présence de la convention industrielle, dans les PVC de type 2, la convention domestique et dans les PVC de type 3 ( «au terroir ») la présence marquée de la convention marchande. 
développement, constitutives des réseaux alimentaires de proximité. Ces coordinations construites autour de la mise à disposition de produits alimentaires organisent de nouvelles stratégies multiformes mises en œuvre par les agriculteurs et les consommateurs, au sein d'une gouvernance de la qualité, à la fois constitutive et effet de la convention de réseau.

\section{RÉFÉRENCES BIBLIOGRAPHIQUES}

Akerlof G. (1970). The Market for lemons: Quality Uncertainty and the Market mechanism. Quarterly Journal of Economics, vol. $84, \mathrm{n}^{\circ} 3$, p. 488-500.

Allaire G. (1995). De la productivité à la qualité, transformation des conventions et régulations dans l'agriculture et l'agroalimentaire. In Allaire G., Boyer R. (éds.) «La grande transformation de l'agriculture », Éditions INRA-Economica.

Aristote (2007). Réédition « Rhétorique ». Présentation et traduction par Chiron P., Paris, G.F. Flammarion.

Aznar O., Bretière G., Herviou S. (2006) Agriculture de services note de synthèse sur l'agriculture de service pour le Ministère de l'Agriculture et de la Pêche.

Agriculture de service et services environnementaux : lien avec les politiques agricoles et rurales (2006) ed. CEMAGREF

Buhler E.-A., Coquart D., Girou S., Fontorbes J.-P., Mondy B., Olivier V., Pilleboue J., Vincq J.-L. (2008). Les relations de proximité agriculteurs-consommateurs : points de vente collectifs et AMAP en Midi-Pyrénées. Toulouse, Dynamiques Rurales, Programme SHS, Dossier n ${ }^{\circ} 06020615,265$ p.

Boltanski L., Chiappello E., (1999.) Le nouvel esprit du capitalisme éd. Gallimard

Cochoy F. (2001). La captation des publics entre dispositifs et dispositions, ou le petit chaperon rouge revisité. Pour une sociologie du travail relationnel. Université de Versailles, Saint-Quentin-en-Yvelines, Journée d'études sur les dispositifs de gestion, 4-5 octobre.

Cochoy F. (2002). Figures du client, leçons du marché. Sciences de la Société, $n^{\circ} 56$, mai.

Delfosse C., Letablier, M.-T., (1995). Genèse d'une convention de qualité. Cas des appellations d'origine fromagère. In « $L a$ grande transformation de l'Agriculture », INRA Economica.

Delpal F., Hatchuel G. (2007). La consommation engagée s'affirme comme une tendance durable. CREDOC, Consommation et mode de vie, $\mathrm{n}^{\circ} 201$, mars.

Dubuisson-Quellier S., Neuville J.-P. (2003). Introduction à Juger pour échanger. De la qualité sur les marchés et dans les organisations. Les modalités de la formation d'un jugement équipé pour l'échange. Éditions de la Maison des sciences de l'homme et Institut National de la Recherche Agronomique.

Dubuisson-Quellier S., et Lamine C., (2003) Pluralité des formes d'engagement des consommateurs sur les marchés : le cas des produits issus du commerce équitable. Communication à GDR Économie et sociologie des marchés agroalimentaires, Montpellier 23 et 24 mars 2006.

Dujarier M.-A. (2008). Le travail du consommateur. De MacDo à Ebay : comment nous coproduisons ce que nous achetons. Paris, La Découverte.

Eymard-Duvernay F. (2004). Coordination des échanges par l'entreprise et qualité des biens. In Orléan A. (dir.) « Analyse économique des conventions », PUF.

Eymard-Duvernay F. (1986). La qualification des produits. In Salais R., Thévenot L. (éds.) «Le travail, Marché , règles, conventions », Paris, INSEE Economica. p. 239-247. 
Eymard-Duvernay F. (1989). Conventions de qualité et formes de coordination. Revue Economique, $\mathrm{n}^{\circ} 2$.

Eymard-Duvernay F., Favereau, O., Orléan, A., Salais, R, Thévenot, (2004). Valeurs, coordination et rationalité ; trois thèmes mis en relation par l'économie des conventions. In «L'économie des conventions : Méthodes et résultats », Paris, Éditions La Découverte.

Foray M. (1995). Standards de référence, coûts de transaction et économie de la qualité : un cadre d'analyse. In Nicolas F., Valceschini E. (éd.) «Agroalimentaire : une économie de la qualité».

Gadrey J. (1996). L'économie des services. Paris, La Découverte, Coll. Repères.

Gomez P.-Y. (1994). Qualité et théorie des conventions, Paris, Economica.

Hebel P. (2005). La santé de plus en plus prégnante dans l'alimentation des Français. CREDOC, Cahiers de Recherche, $\mathrm{n}^{\circ} \mathrm{C} 186,09 / 2005$.

Hérault-Fournier C., Prigent-Simonin A.-H. (2005). La dimension relationnelle de la qualité des produits alimentaires. Communication au symposium international «Territoires et enjeux du développement régional », Lyon, 9-11 mars.

Karpik L. (1989). L'économie de la qualité. Revue Française de Sociologie XXX, $\mathrm{n}^{\circ} 2$, p. 187-210.

Karpik L. (1996). Dispositifs de confiance et engagements crédibles. Sociologie $d u$ travail, $\mathrm{n}^{\circ} 4 / 96$.

Karpik L. (2007). « L'économie des singularités ». Paris, Gallimard.

Knight F. (1921). Risk, uncertainty and profit. Boston, Edition Houghton Mifflin.

Kreps D.-M. (1990). Corporate Culture and Economic Theory. In Alt J., Shepsle K. (dirs.) "Perspective on positive political economy", Cambridge, Cambridge University Press.

Lagrange L., Valceschini E. (2007). L'économie de la qualité : enjeux, acquis et perspectives. Économie Rurale, n 299 , mai-juin.
Mangematin V. (2003). Cléopâtre et son goûteur. In « Des mondes de confiance, un concept à l'épreuve de la réalité sociale », sous la direction de Mangematin V. Thuderoz C., CNRS Éditions.

Mangematin V., Thuderoz C. (2003). Conclusion à l'ouvrage collectif Des mondes de confiance, CNRS Éditions.

Nefussi J. (2005), Tertiarisation des filières et reconstruction du sens à travers des récits collectifs. Exemples de l'industrie informatique et de la viande porcine, Revue Française de Gestion.

Orléan A. (2004a ) Analyse économique des conventions. Paris, PUF, $2^{\mathrm{e}}$ édition.

Orléan A. (2004b) Vers un modèle général de la coordination économique par les conventions. In Analyse Éonomique des Conventions, Paris, PUF, $2^{\mathrm{e}}$ édition.

Nobecourt J.-P. (1998). Hommes territoires produits. La nouvelle donne agricole. Actes du colloque Salon International de l'Agriculture.

Rallet A., Torre A. (2004). Proximité et localisation. Économie rurale, $\mathrm{n}^{\circ} 280$, mars-avril.

Salais R. (2004). Incertitude et interactions de travail : des produits aux conventions. In Orléan A. (dir.) «Analyse économique des conventions $»$, Paris, PUF, $2^{\mathrm{c}}$ édition.

Rochefort, R. (1997), Le consommateur entrepreneur : nouveaux modes de vie, éd. Odille Jacob, 302 p.

Sauvée L., Valceschini E. (2003). Agroalimentaire : la qualité au cour des relations entre agriculteurs, industriels et distributeurs. Paris, Amand Collin, Démeter 2004, p. 181-226.

Sylvander B. (1995). Conventions de qualité, concurrence et coopération. Cas du « label rouge » dans la filière volailles. In « $l a$ grande transformation de l'agriculture », INRA Economica, p. 73-97.

Zucker L. (1996). Production of trust: Institutional Sources of Economic Structure (1840-1920). Research in Organisation Behaviour, vol. 8, p. 53-111. 